\title{
Macrophages and Thrombin-Another Link between Inflammation and Coagulation
}

\author{
Johann Wojta ${ }^{1}$ \\ ${ }^{1}$ Department of Internal Medicine II, Medical University of Vienna, \\ Vienna, Austria
}

Thromb Haemost 2020;120:537.

Macrophages are highly versatile cells of the innate immune system with a high degree of plasticity. In response to their respective tissue environment they can adopt various functionally different phenoytpes or polarization states. In response to inflammatory stimuli macrophages polarize into proinflammatory M1 cells whereas anti-inflammatory stimuli induce alternative polarization into anti-inflammatory, reparative M2 macrophages. Accumulating evidence supports the notion that macrophages represent a cellular link between inflammation and cardiovascular pathologies such as atherogenesis and thrombosis. ${ }^{1}$ On the other hand, thrombin is not only the central protease in the coagulation cascade but among various other functions also modulates inflammatory processes. ${ }^{2}$ In this issue of Thrombosis and Haemostasis, López-Zambrano et al have revealed yet another link between the coagulation system and inflammation modulated by cells of the innate immune system. ${ }^{3}$ The authors show in vitro that thrombin induces macrophages to polarize toward an M1 phenotype that is characterized by the expression of proinflammatory cytokines and chemokines. This effect of thrombin was at least in part mediated via protease activated receptor-1 (PAR-1). Interestingly, heat denatured, enzymatically inactive thrombin was still effective in inducing a proinflammatory response in macrophages suggesting a pathway independent of PAR-1, which has to be proteolytically cleaved to induce signal transduction. Taken together, this study provides evidence that in a prothrombotic microenvironment, formed thrombin is not only able to
Address for correspondence Johann Wojta, PhD, Department of Internal Medicine II, Medical University of Vienna, Waehringer Guertel 18-20, Vienna 1090, Austria

(e-mail: johann.wojta@meduniwien.ac.at).

promote thrombus formation but also can shift the setting toward inflammation by affecting the polarization state of macrophages. Interestingly, proinflammatory M1 macrophages in contrast to anti-inflammatory M2 macrophages are proteolytically highly active. ${ }^{4}$ The kinetics and dynamics of macrophage polarization toward a particular phenotype have mainly been assessed in in vitro studies. Thus, future investigations addressing these research questions in vivo are highly relevant.

\section{Conflict of Interest}

None declared.

\section{References}

1 Liberale L, Dallegri F, Montecucco F, Carbone F. Pathophysiological relevance of macrophage subsets in atherogenesis. Thromb Haemost 2017;117(01):7-18

2 Posma JJ, Grover SP, Hisada Y, et al. Roles of coagulation proteases and PARs (protease-activated receptors) in mouse models of inflammatory diseases. Arterioscler Thromb Vasc Biol 2019;39 (01):13-24

3 López-Zambrano M, Rodriguez-Montesinos J, Crespo-Avilan GE, Muñoz-Vega M, Preissner KT. Thrombin promotes macrophage polarisation into M1-like phenotype to induce inflammatory responses. Thromb Haemost 2020;120(04):658-670

4 Hohensinner PJ, Baumgartner J, Kral-Pointner JB, et al. PAI-1 (plasminogen activator inhibitor-1) expression renders alternatively activated human macrophages proteolytically quiescent. Arterioscler Thromb Vasc Biol 2017;37(10):1913-1922 received

February 24, 2020

accepted after revision

February 24, 2020 (c) 2020 Georg Thieme Verlag KG Stuttgart · New York
DOI https://doi.org/ 10.1055/s-0040-1708551. ISSN 0340-6245. 